\title{
Fundamental groups of iterated line graphs
}

\author{
E. H. Hamouda ${ }^{1 *}$, M. S. Fahmy ${ }^{2}$ \\ ${ }^{1}$ Department of basic sciences, faculty of industrial education, Beni-Suef university, Egypt \\ ${ }^{2}$ Faculty of engineering, modern sciences and arts university, Egypt \\ *Corresponding author E-mail: ehamouda70@gmail.com
}

Copyright $\odot 2014$ E. H. Hamouda, M. S. Fahmy. This is an open access article distributed under the Creative Commons Attribution License, which permits unrestricted use, distribution, and reproduction in any medium, provided the original work is properly cited.

\begin{abstract}
The rank of the fundamental group, $\pi(G)$, of a connected graph $G$ is related to the Euler characteristic, $\chi(G)$, of $G$ by $\pi(G)=1-\chi(G)$. in this article, the Euler characteristic of the $i$ th iterated line graph of $G$ and its complement $\bar{G}$ is studied.

Keywords: line graphs, iterated line graphs, fundamental groups.

\section{Introduction}

We follow [2] for graph theoretical terminologies and notations that are not defined here. Graphs considered in this paper are finite and simple connected graphs (without loops and parallel edges). In general, $V_{G}$ refers to the set of vertices of a graph $G$, and $E_{G}$ refers to the edges of $G$. The number of vertices and edges of $G$ are denoted by $\left|V_{G}\right|$ and $\left|E_{G}\right|$ respectively. The fundamental group is a much studied topic in elementary topology. As graphs are also topological spaces [4], many authors investigated the fundamental group structure of an arbitrary graph $G$ ([5], [6], [8], [10]). If $G$ is a connected graph has $|V|$ vertices and $|E|$ edges, the number $\pi(G)=1-|V|+|E|$ is the rank of the fundamental group of $G[6]$ and is related to the Euler characteristic, $\chi(G)$, of $G$ by $\pi(G)=1-\chi(G)$ [4]. This value equals the Betti number $\beta(G)$, which is nonnegative for connected $G$ and was one of the first numerical characteristics of a graph. Also some connections between the fundamental group of a graph, the genus of the graph, and the number of components of a 2-manifold in which $G$ can be embedded are introduced in [3]. In [1], the number $\pi(G)$ was defined as the number of independent cycles for some $G$. In this article, the Euler characteristic of the $i$ th iterated line graph of $G$ and its complement $\bar{G}$ is studied.
\end{abstract}

\section{Euler characteristic of $L(G)$}

For a graph $G$, the line graph $L(G)$ is a graph whose vertices can be put in a one-to-one correspondence with the edges of $G$, in such a way that two vertices in $L(G)$ are adjacent if and only if the corresponding edges of $G$ are adjacent. The concept has been rediscovered several times, with different names such as derived graph, interchange graph [9, 11], and edge-to-vertex dual. We iterate the line graph of $G$ in the natural way by setting $L^{i}(G)=L\left(L^{i-1}(G)\right)$, where $L^{0}(G)=$ $G$. If $d(v)$ is the degree of a vertex $v$ and $\left|V_{G}\right|,\left|E_{G}\right|,\left|V_{L}\right|,\left|E_{L}\right|$ denote the number of vertices and edges of $G$ and $L(G)$ respectively, then clearly, $\left|V_{L}\right|=\left|E_{G}\right|$ and it is well known that [1]

$$
\begin{aligned}
\left|E_{L}\right| & =\sum_{v \in V_{G}} \frac{d(v)(d(v)-1)}{2} \\
& =\frac{1}{2} \sum_{v \in V_{G}} d(v)^{2}-\frac{1}{2} \sum_{v \in V_{G}} d(v) \\
& =\frac{1}{2} \sum_{v \in V_{G}} d(v)^{2}-\left|E_{G}\right|
\end{aligned}
$$


Now for $L(G)$ we have

$$
\begin{aligned}
\chi(L(G)) & =\left|V_{L}\right|-\left|E_{L}\right| \\
& =\left|E_{G}\right|-\left(\frac{1}{2} \sum_{v \in V_{G}} d(v)^{2}-\left|E_{G}\right|\right) \\
& =2\left|E_{G}\right|-\frac{1}{2} \sum_{v \in V_{G}} d(v)^{2}
\end{aligned}
$$

For a regular graph $G$ of degree $r$, every vertex $v \in V_{L}$ corresponding to an edge $e=x y \in E_{G}$ has degree equals $d(x)+d(y)-2$. Thus $L(G)$ is regular of degree $2 r-2$.

We put the following facts in the form of a lemma, which comes immediately from the definition of Euler characteristic of $G$.

Lemma 2.1: Let $G$ be a $r$-regular graph with $n$ vertices, then

a) $\chi(G)=\frac{n(2-r)}{2}$.

b) $\chi(L(G))=r \chi(G)$.

Lemma 2.2: Let $G$ be a $r$-regular graph with $n$ vertices, then $\chi\left(L^{2}(G)\right)=(2 r-2) \chi(L(G))$.

Proof: Since $L(G)$ is a $(2 r-2)$-regular graph with $\frac{n r}{2}$ vertices. By equation $(1),\left|E_{L(G)}\right|=\frac{1}{2}\left[n r^{2}\right]-\frac{n r}{2}=\frac{n r}{2}(r-1)$. Therefore, $L^{2}(G)$ is a regular graph contains $\left|E_{L(G)}\right|$ vertices of degree $4 r-6$. Hence, by lemma 2.1 , we conclude that $\chi\left(L^{2}(G)\right)=\frac{1}{2}\left[\frac{n r}{2}(r-1)(8-4 r)\right]=\frac{n r(2 r-2)(2-r)}{2}=(2 r-2) \chi(L(G))$

Now we move on to generalize lemma 2.2. Let $G$ be a $r$-regular graph with $n$ vertices. For $i>0$, assume $r_{i}$ denotes the degree of the regular graph $L^{i}(G)$ such that $r_{i}=2 r_{i-1}-2$, and $r_{0}=r$.

Theorem 2.3: $\chi\left(L^{i}(G)\right)=r_{i-1} \cdot \chi\left(L^{i-1}(G)\right)$.

Proof: Straightforward.

\section{Euler characteristic of $\bar{G}$}

The complement $\bar{G}$ of a graph $G$ has the same vertices as $G$, and every pair of vertices is joined by an edge in $\bar{G}$ if and only if they are not joined in $G$. It is known that $\bar{G} \cup G=K_{n}$, but this is not enough to say $\chi\left(K_{n}\right)=\chi(G)+\chi(\bar{G})$. a self-complementary graph $G$ is one that is isomorphic to its complement.

The following results are straightforward, and are not stated explicitly by any author. However, they are all useful in proving other results. For a complete graph $K_{n}$ and $e \in E_{K_{n}}$, we define $L(e)$ to be a vertex $v \in V_{L}$ of degree $d(v)=$ $2(n-1)-2$.

Proposition 3.1: Let $G=K_{n}-e$, then $L(G)=L\left(K_{n}\right)-L(e)$.

Proof: Notice that $G$ has $n-2$ vertices of degree $n-1$ and 2 vertices of degree $n-2$. Hence, $L(G)$ has $\frac{n(n-1)}{2}-1$ vertices and $\left|E_{L}\right|$ edges comes by equation (1) as follows:

$$
\begin{aligned}
\left|E_{L}\right|=\frac{1}{2} \sum_{v \in V_{G}} d(v)^{2}-\left|E_{G}\right| & =\frac{1}{2}\left[(n-2)(n-1)^{2}+2(n-2)^{2}\right]-\left[\frac{n(n-1)}{2}-1\right] \\
=\frac{1}{2}\left[(n-2)\left(n^{2}-3\right)\right]-\frac{1}{2}\left(n^{2}-n-2\right)=\frac{1}{2}\left(n^{3}-3 n^{2}-2 n+8\right) & \\
= & \frac{1}{2}(n-2)\left(n^{2}-n-4\right)=(n-2)\left[\frac{n(n-1)}{2}-2\right] \\
= & \frac{1}{2}[n(n-1)(n-2)]-2(n-2) .
\end{aligned}
$$

By lemma 2.1, this becomes

$$
\left|E_{L(G)}\right|=\left|E_{L\left(K_{n}\right)}\right|-d(v) .
$$

This implies, $L(G)=L\left(K_{n}\right)-L(e)$. $\square$ 
We say that a tree $T$ is a spanning subgraph of $K_{n}$ if $\left|V_{T}\right|=n$ [2]. Let $\bar{T}=K_{n}-T$ be obtained by deleting all edges of

$T$. It is obvious that, $\bar{T}$ may be has isolated vertices when $d(v)=n-1$ for some $v \in V_{T}$.

Theorem 3.2: Let $T$ be a spanning tree in $K_{n}$ such that $d(v) \leq 2$ for all $v \in V_{T}$. Then

$$
\chi(L(\bar{T}))=\frac{(n-2)\left(-n^{2}+6 n-7\right)}{2} .
$$

Proof: Since $d(v) \leq 2$ for all $v \in V_{T}$, then $\bar{T}=K_{n}-T$ have $\frac{(n-1)(n-2)}{2}$ vertices, $n-2$ of them of degree $n-3$ and two vertices of degree $n-2$. By equation (1), we have

Now, we conclude that

$$
\begin{aligned}
\left|E_{L(\bar{T})}\right|= & \frac{1}{2}\left[(n-2)(n-3)^{2}+2(n-2)^{2}\right]-\left[\frac{n(n-1)}{2}-(n-1)\right] \\
& =\frac{1}{2}\left[(n-2)\left(n^{2}-4 n+5\right)\right]-\frac{1}{2}[(n-1)(n-2)] \\
& =\frac{1}{2}\left[(n-2)\left(n^{2}-5 n+6\right)\right]=\frac{1}{2}\left[(n-3)(n-2)^{2}\right] .
\end{aligned}
$$

$$
\chi(L(\bar{T}))=\frac{(n-1)(n-2)}{2}-\frac{(n-3)(n-2)^{2}}{2}=\frac{(n-2)\left(-n^{2}+6 n-7\right)}{2} . \square
$$

Let $H$ be an induced subgraph of $K_{n}$, and $G=K_{n}-H$ be obtained by deleting all edges of $H$. A graph $G$ that has some isolated vertices $x$ and is therefore disconnected, may nevertheless have a connected line graph. So, we assume that $d(v)<n-1$ for all $v \in V_{H}$, the following theorem generalizes the previous result.

Theorem 3.3: Let $G=K_{n}-H$. Then

$$
\begin{aligned}
& \text { a) }\left|V_{L(G)}\right|=\left|V_{L\left(K_{n}\right)}\right|-\left|V_{L(H)}\right| . \\
& \text { b) }\left|E_{L(G)}\right|=\left|E_{L\left(K_{n}\right)}\right|+\left|E_{L(H)}\right|-(2 n-4)\left|E_{H}\right| .
\end{aligned}
$$

Proof: (a) Since $H$ is a complete subgraph [2]. Then, we assume $H$ has $r<n$ vertices and $\frac{r(r-1)}{2}$ edges. It follows that $K_{n}-H$ contains $n-r$ vertices of degree $n-1$ and $r$ vertices of degree $[(n-1)-(r-1)]$. therefore, $L\left(K_{n}-H\right)$ has $\left[\frac{n(n-1)}{2}-\frac{r(r-1)}{2}\right]$ vertices.

(b) By equation (1), we get

By lemma 2.1, we get

$$
\begin{aligned}
\left|E_{L\left(K_{n}-H\right)}\right| & =\frac{1}{2}\left[(n-r)(n-1)^{2}+r(n-r)^{2}\right]-\left[\frac{n(n-1)}{2}-\frac{r(r-1)}{2}\right] \\
& =\frac{1}{2}\left[n^{3}-2 n^{2}+n+2 r n-r-2 r^{2} n+r^{3}\right]-\frac{1}{2}\left[n^{2}-n-r^{2}+r\right] \\
& =\frac{1}{2}\left[n^{3}-3 n^{2}+2 n+2 r n-2 r-2 r^{2} n+r^{2}+r^{3}\right] \\
& =\frac{1}{2}\left[n^{3}-3 n^{2}+2 n\right]+\frac{1}{2}\left[r^{3}-3 r^{2}+2 r\right]-\frac{1}{2}\left[2 r^{2} n-2 r n-4 r^{2}+4 r\right] \\
& =\frac{1}{2}\left[n^{3}-3 n^{2}+2 n\right]+\frac{1}{2}\left[r^{3}-3 r^{2}+2 r\right]-\frac{1}{2}[r(r-1)(2 n-4)] .
\end{aligned}
$$

$$
\left|E_{L(G)}\right|=\left|E_{L\left(K_{n}\right)}\right|+\left|E_{L(H)}\right|-(2 n-4)\left|E_{H}\right| . \square
$$

From the preceding discussion and theorem 3.3, we summarize with.

Theorem 3.4: Let $G$ be a graph (may be disconnected) with $n$ vertices such that $d(v)<n-1$ for all $v \in V_{G}$. Then
a) $\left|V_{L(\bar{G})}\right|=\left|V_{L\left(K_{n}\right)}\right|-\left|V_{L(G)}\right|$.
b) $\left|E_{L(\bar{G})}\right|=\left|E_{L\left(K_{n}\right)}\right|+\left|E_{L(G)}\right|-(2 n-4)\left|E_{G}\right|$.

\section{Some applications}

The maximum genus of a connected graph $G$, denoted by $\gamma_{M}(G)$, is the maximum integer $k$ with the property that there exists a cellular embedding of $G$ on the orientable surface with genus $k$. The maximum genus of many kinds of graphs in terms of some graph invariants such as connectivity, diameter, girth, and chromatic number and The Betti number 
$\beta(G)$ are investigated [5]. In theory, the deciding problem of genus of a graph is always difficult [Deciding the genus of a graph is NP-complete, 5]. Authors in [6], studied the relations between the maximum genus and the matching number and they showed that they are coincident for some graphs. In [14], lower bounds on the maximum genus of connected 4-regular simple graphs and connected 4-regular graphs without loops are calculated in terms of the Betti number. Since The Betti number $\beta(G)$ equals the rank of the fundamental group $\pi(G)$, so lower bounds on the maximum genus of graphs may be obtained in terms of $\pi(G)$.

By lemma 2.1, the following corollary comes directly from theorem A in [14].

Corollary 4.1: If $G$ is a connected 4-regular simple graph with $n$ vertices, then

$$
\gamma_{M}(G) \geq\left[1+\frac{2 n}{5}\right]
$$

Authors in [13], proved the next result for $i=1$. We consider the general case as an application of the above results.

Corollary 4.2: $L^{i}\left(K_{n}\right) \cong K_{n}$ implies that $\left|E_{L^{i}}\right|=\left|V_{L^{i}}\right|$.

Proof: We use induction on $i$. when $i=1$, suppose $L^{1}\left(K_{n}\right) \cong K_{n}$, this implies that $\chi\left(L\left(K_{n}\right)\right)=\chi\left(K_{n}\right)$. From lemma 2.1, we have $\frac{n(n-1)(3-n)}{2}=\frac{n(3-n)}{2}$. Thus the number of vertices in $K_{n}$ equals 2, i.e. $n=2$, this means that the complete graph must be $K_{2}$. Hence, $\left|E_{L^{1}}\right|=\left|V_{L^{1}}\right|$. Suppose by the inductive hypothesis that, $L^{i-1}\left(K_{n}\right) \cong K_{n}$ implies that $\left|E_{L^{i-1}}\right|=\left|V_{L^{i-1}}\right|$. Assume that $L^{i}\left(K_{n}\right) \cong K_{n}$. Then $\chi\left(L^{i}\left(K_{n}\right)\right)=\chi\left(K_{n}\right)$ and by theorem 2.3 , we have $\left|E_{L^{i}}\right|-$ $\left|V_{L^{i}}\right|=r_{i-1} \chi\left(L^{i-1}\left(K_{n}\right)\right)=r_{i-1}\left(\left|E_{L^{i-1}}\right|-\left|V_{L^{i-1}}\right|\right)$. But $\left|E_{L^{i-1}}\right|=\left|V_{L^{i-1}}\right|$ yields the desired result. $\square$

It is well known that the fundamental group of a graph $G$ is trivial if and only if $G$ is a tree [4]. The following result is a direct application of theorem 3.2.

Corollary 4.3: Let $T$ be a spanning tree in $K_{n}$ Such that $d(v) \leq 2$ for all $v \in V_{T}$, then $L(\bar{T})$ is a tree if and only if $n=1,2,3$.

Proof: Assume that $L(\bar{T})$ is a tree, then $\chi(L(\bar{T}))=\frac{(n-2)\left(-n^{2}+6 n-7\right)}{2}=1$. Simple counting arguments shows that $n=1,3,4$. This means $L(\bar{T})$ is a tree only in case of $K_{n}$ is a vertex, a triangle or $K_{4}$. $\square$

Corollary 4.4: If $G$ is a self-complementary graph, then $\left|E_{G}\right|=\frac{\left|V_{G}\right|\left(\left|V_{G}\right|-1\right)}{4}$.

Proof: Since $G$ is isomorphic to $\bar{G}$, then $L(\mathrm{G})$ is isomorphic to $L(\bar{G})$. By part (a) of theorem 3.4, we have

$$
\left|V_{L(G)}\right|=\left|E_{G}\right|=\left|V_{L(\bar{G})}\right|=\left|V_{L\left(K_{n}\right)}\right|-\left|V_{L(G)}\right| \text {. }
$$

This becomes, $\left|E_{G}\right|=\frac{\left|V_{G}\right|\left(\left|V_{G}\right|-1\right)}{2}-\left|E_{G}\right|$. Hence, we have

$$
\left|E_{G}\right|=\frac{\left|V_{G}\right|\left(\left|V_{G}\right|-1\right)}{4} \text {. }
$$

Corollary 4.5: Let $G$ be a regular graph of degree $r$ with $n$ vertices. If $L(G) \cong L(\bar{G})$ then $G \cong C_{5}$.

Proof: Suppose $G$ consists of $k$ components, then clearly $L(G)$ also has $k$ components. But $\bar{G}$ consists of only one component; hence we must have $k=1$, i.e., $G$ is a connected graph. Since $L(G) \cong \bar{G}$ implies that $\chi(K(G))=\chi(\bar{G})$. Then we get $\frac{n r(2-r)}{2}=\left[n-\left(\frac{n(n-1)}{2}-\frac{n r}{2}\right)\right]$. This become $r(1-r)=3-n$. Since there is no connected graph consists of 3 vertices with degree one. Therefore, we consider $r>1$ and consequently $n>3$. Since $L(G) \cong \bar{G}$ means that $\left|V_{L(G)}\right|=\frac{n r}{2}=\left|V_{\bar{G}}\right|=n$; hence we must have $r=2$. We conclude that $n=5$ and $G \cong C_{5}$ are the only possible 2-regular graph in this case. $\square$

\section{References}

[1] M. Aigner, "Graphs whose complement and line graph are isomorphic", J. of Combinatorial Theory 7 (1969) $273-275$.

[2] J. Bondy, U. Murty, Graph theory with applications, New York: Macmillan; 1976.

[3] R. Ducke, "How is a graph's Betti number related to its genus?", The American Mathematical Monthly, Vol. 78, No. 4. (1971) 386-388.

[4] A. Hatcher, Algebraic topology, Cambridge University Press; 2001.

[5] R. Han, L. Gang, "Survey of maximum genus of graphs", J. of East China Normal University, No. 5 (2010), 1 - 19.

[6] M. Kotrbeik, M. Skoviera, Matchings, "cycle bases, and the maximum genus of a graph", the electronic j. of combinatorics 19(3) (2012), \#P3. 
[7] F. Larrión, M. Pizaña, R. Villarroel-Flores, "The fundamental group of the clique graph", European J. Combin. 30 (2009) $288-294$.

[8] M. Imbert, "Fundamental groups of fat graphs", Note di Matematica Vol. 19, No. 2(1999) 257-268.

[9] W. Massy, Algebraic topology: An introduction. Harcourt, Brace \& World, New York; 1967.

[10] S. Nada, E. H. Hamouda, "Fundamental group of dual graphs and applications to quantum space-time", Chaos, Solitons and Fractals 42 (2009) 500-503.

[11] B. Schwartz, "On Interchange Graphs", pacific J. of Mathematics, Vol. 27,No. 2.; (1968) 393-396.

[12] J. Stallings, "Topology of Finite Graphs", Invent. Math. 71, 551-565 (1983).

[13] A. van Roij, H. Wilf, "The Interchange Graph of a finite graph", Acta. Math. 16, (1983) 263-269.

[14] D. Zhou, R. Hao, W. He, "Lower bounds for the maximum genus of 4-regular graphs", Turk J Math 36 (2012), 530 - 537. 